\title{
Review on Characterization of Cowpea Germplasm in Terms of Distinctness, Uniformity, Stability and Novelty for Morphological, Quality and Yield Attributing Parameters
}

\author{
Sanjeev K. Deshpande ${ }^{1^{*}}$, B.R. Mani ${ }^{2}$, S.A. Desai ${ }^{3}$, \\ T.K. Nagarathna ${ }^{3}$ and R.R. Hanchinal ${ }^{3}$ \\ ${ }^{1}$ G.P.B., University of Agricultural Sciences, Dharwad, Karnataka-580 005, India \\ ${ }^{2}$ SRF, Cowpea DUS, University of Agricultural Sciences, Dharwad, Karnataka-580 005, India \\ ${ }^{3}$ PPV \& FRA, IARI, New Delhi-110 012, India
}

\begin{tabular}{|l|}
\hline Ke y w o r d s \\
$\begin{array}{l}\text { DUS test, Novelty, } \\
\text { germplasm, Pulses, } \\
\text { Characteristics }\end{array}$ \\
\hline Article Info \\
\hline $\begin{array}{l}\text { Accepted: } \\
\text { 06 May } 2018 \\
\text { Available Online: } \\
\text { 10 June } 2018\end{array}$ \\
\hline
\end{tabular}

\section{Introduction}

The cowpea (Vigna unguiculata) is an annual herbaceous legume of the genus Vigna. Due to its tolerance for sandy soil and low rainfall it is an important crop in the semi-arid regions across India and other countries. It requires very few inputs, as the plants root nodules are
Cowpea (Vigna unguiculata L.) is the most important grain legume crop grown in tropical and subtropical regions of the world. In India cowpea is grown as sole, mix-crop, intercrop systems. Cowpea is however, the only pulse suited to both arid and semi-arid regions and adapted better than any other pulses in high rainfall regions. As a good source of proteins, calcium and iron, it is recognized for many routine uses of food, feed, forage, fodder, vegetable and a number of dishes prepared from its grains. India being a secondary centre of diversity for cowpea, possess huge germplasm and wild relatives of cowpea thus, it supplements to the genetic resources of worldwide cowpea collection. Characterization of germplasm became mandatory for varietal registration and to apply for a plant breeder right. The characterization data on various morpho-physiological and agronomic traits is essential to maintain genetic data base at seed banks. In this context, a review has been conducted for the literature regarding DUS characterization, its importance and method of experimentation, various descriptors and documentation of data. The data available in the literature has been utilized in classification of cowpea plant characters into ten distinct categories, considering the seedling, stem, leaf, flower, pod, seed and plant morphological traits. Further, under each category, distinguishable, unique and stable traits were also been formulated as DUS parameters of cowpea. The present review may help the researchers in understanding the scope of DUS test and methodology of experimentation moreover, its present day importance with respect to germplasm conservation and patents. 
diversity found within the species with large variations in the size, shape and structure of the plant. Cowpeas can be erect, semi erect (trailing) or climbing. The crop is mainly grown for its seeds, which are extremely high in protein, although the leaves and immature seed pods can also be consumed.

Cultivated cowpeas are known by the common names black-eyed pea, southern pea, yard long bean, catjang, and crowder pea.

They were domesticated in Africa and are one of the oldest crops to be farmed. A second domestication event probably occurred in Asia, before they spread into Europe and the Americas. The seeds are usually cooked and made into stews and curries, or ground into flour or paste.

The Indian subcontinent is very rich in biological diversity, harboring around 49,000 species of plants, including about 17,500 species of higher plants. The Indian gene centre holds a prominent position among the 12 mega-gene centers of the world. It is also one of the Vavilovian centers of origin and diversity of crop plants. Two out of the 25 global hotspots of biodiversity, namely the Indo-Burma and Western Ghats are located here.

India possesses about 12 per cent of world flora with 5725 endemic species of higher plants belonging to about 141 endemic genera and over 47 families. About 166 species of crops including 25 major and minor crops have originated and/or developed diversity in this part of the world. Further, 320 species of wild relatives of crop plants are also known to occur here. Presently, the Indian diversity is composed of rich genetic wealth of native as well as introduced types. It is also a secondary centre of diversity for African crops like finger millet, pearl millet, sorghum, cowpea, cluster bean, okra, sesame, niger and safflower

\section{Crop description}

There is a large morphological diversity found within the crop, and the growth conditions and grower preferences for each variety vary from region to region (Padulosil, 1997). However, as the plant is primarily self-pollinating its genetic diversity within varieties is relatively low. Cowpeas can either be short and bushy (as short as $20 \mathrm{~cm}$ ) or act like a vine by climbing supports or trailing along the ground (to a height of $2 \mathrm{~m}$ ) (Sheahan, 2012) and National Research Council (2006). The tap root can penetrate to a depth of $2.4 \mathrm{~m}$ after eight weeks of sowing (Egbadzor et al., 2014). The size and shape of the leaves varies greatly, making this an important feature for classifying and distinguishing cowpea varieties (Pottorff, et al., 2012). Another distinguishing feature of cowpeas is the long 20-50 $\mathrm{cm}$ peduncles which hold the flowers and seed pods. One peduncle can support four or more seed pods (Davis, et al., 1991). Flower color varies through different shades of purple, pink, yellow and white and blue (National Research Council, 2006). Seeds and seed pods from wild cowpeas are very small (Davis et al., 1991). While, cultivated varieties can have pods between 10 and $110 \mathrm{~cm}$ long (Rawal and Kanti 1975). A pod can contain 613 seeds that are usually kidney shaped, although the seeds become more spherical the more restricted they are within the pod (Davis, et al., 1991). Their texture and color is very diverse. They can have a smooth or rough coat, and be speckled, mottled or blotchy. Colors include white, cream, green, red, brown and black or various combinations (Davis, et al., 1991).

\section{Classification of cowpea varieties based on its bean shape, size and color}

(Source: India Pulses and Grains Association, www.nutritiondata.self.com, www.nda.agric.za) 


\section{Black-eyed or pink-eyed/purple hull peas}

These white colored seeds have black, pink or purple eye like shape around the hilum. Seeds are not tightly packed or crowded in the pod and are kidney shaped or oblong.

\section{Brown-eyed peas}

These types of cowpeas have green to lavender colored pods. When immature seeds of this type are cooked, it has very tender and a delicate flavor.

\section{Crowder peas}

As the name suggests, this type of cowpeas are "crowded" in the pod and have seed of black, speckled and brown eyed.

\section{Cream peas}

Seeds are cream colored. This type of seeds looks like intermediate between the blackeyed and crowder types.

\section{White acre type}

Seeds are kidney-shaped with a blunt end, semi crowded and generally tan in color.

\section{Clay types}

These older varieties are medium to dark brown in color and kidney- shaped, but are rarely grown

\section{Importance of germplasm resources in crop improvement}

In order to breed qualities of resistance, adaptation and better nutritive value into crops, breeders need sources of genetic diversity to draw upon when required. This diversity is generally spoken of as 'genetic resources' or 'gene pools'. These gene pools cannot be created artificially except to a limited extent through mutation breeding or genetic engineering. Fortunately, a large reservoir of genetic diversity existed until recently in old 'land races' or farmers' varieties; a great deal of useful variation also occurs in related wild species.

Modern agricultural technology and the introduction of new high-yielding varieties are largely eliminating the wide range of crop genetic diversity that has evolved during the five to ten thousand years since food plants were first domesticated. Related wild species are also on the decline because of new land use policies. These gene pools (or what is left of them) are generally spoken of as genetic resources, and are vitally needed in the creation of new crop varieties by plant breeders. Wild species and land races often furnish genes conferring resistance to diseases and pests and adaptation to environmental stresses which cannot be found in the modern crop varieties.

\section{DUS characterization studies in cowpea}

To sustain high production and productivity of cowpea, a considerable number of varieties have been developed. In addition, a number of germplasm collections are available in the gene pool for use in plant breeding programmes. However, there is lack of compilation of key diagnostic characters of these varieties and germplasm collections, which is essential to carryout scientific seed production and certification, endorse proper quality control, DUS testing and utilization of these characteristics in further breeding programmes.

The variety characterization and purity assessment are very important for maintenance, multiplication, seed certification and quality control of released varieties. The characterization is also essential for 
registration and protection of varieties. Traditionally varieties were identified on the basis of their morphological characters, either in the form of seed, seedlings or growing plant characteristics.

In this context, Sarutayophat et al., (2007), characterized 13 cowpea accessions based on growth habit, days to 50 per cent flowering, pod color, pod length, number of pods per plant, seed yield per plant. Similarly, Basavaraj, et al., 2013, conducted an experiment to characterize 35 cowpea genotypes using different quantitative descriptors at plant level. Highly significant differences were obtained among the genotypes for the characters studied. All characters were found stable and reliable for classification except for characters number of pods per peduncle and seed yield per hectare. Hence, it's reliable to consider these stable characters in future breeding programmes of cowpea crop and quality seed production and also in protection in plant variety.

Further, Stoilova and Pereira (2013), had used 24 different morphological descriptors in order to identify accessions with specific behavior that could be exploited by plant breeders and they found that descriptors like pod length, number of seeds per pod, seed thickness and 100 seed weight were the most stable traits and they concluded these characters can be used in characterization.

Sunil Kumar et al., (2015), characterized 20 forage cowpea genotypes on the basis of various morphological characters which were mentioned in cowpea germplasm catalogue of IITA, Nigeria. All genotypes showed indeterminate type of main stem and raceme type of layer in canopy; whereas genotypes viz., KBC 2, IC 249141, HC 46, CO 4, EC 3941-1, EC 101980, Kohinoor and CO 5 showed unique state of expression among nine characters viz., incidence of cowpea mosaic virus, seed crowding in pod, texta structure, eye color, pod shape, pod attachment to peduncle, terminal leaflet shape, twining tendency and flower pigmentation, respectively.

\section{DUS characterization studies in related pulse (Green gram)}

The literature pertaining to DUS characterization in Cowpea is limited, a review is also been made by considering the green gram, a crop relative of cowpea. Rupinder et al., (2017) characterized five lines released by PAU, Ludhiyana (PAU911, SML668, ML818, ML613 and SML832) using morphological and molecular markers.

Phenotypically these varieties showed variation for growth habit, leaf and flower characters, pod color, position and length, plant height, seed coat lusture and seed size during different growth stages of the crop. Plant morphology characters being polygenic in nature are liable to be influenced by the environment. Hence there is a need to use alternate descriptors which are rapid, accurate and less affected by environment.

\section{Methodology of DUS testing}

The principles and methods on various aspects of DUS testing of new crop varieties have been documented as National Test Guidelines (NTG). NTG has been developed for 35 crops by the National Core Committee constituted by ICAR. NTG contains details on plant material required, conduct of tests, methods and observations, grouping of varieties, characteristics and symbols, table of characteristics, literature and technical questionnaire. Usually the DUS examination requires at least two independent growing cycles. Not less than two centers have been identified for each crop for conduction of the tests. 


\section{Distinct-Uniform-Stable (DUS)}

Distinct means a variety should be clearly distinguishable by one or more essential characteristics from any other existing variety. The variety is deemed Uniform if it is sufficiently uniform in its relevant characteristics, subject to variation that may be expected from the particular features of its population.

The basis of assessment is normally the number of off-types in the variety, judged on the basis of a population standard and an acceptable probability fixed in the corresponding species. To identify off-types in a population, generally visual observation on characteristics may suffice.

However, in a few crops, it may be necessary to make measurements of each plant to apply statistics to decide or not whether a plant is an off-type. In most of the crops, acceptance probability of $95 \%$ has been suggested. The variety is said to be Stable if its relevant characteristics remain unchanged after repeated propagation. Though it is not usually possible to assess stability with in a period of 2 or 3 years, the variety can be considered stable if is shown to be uniform.

\section{Characterization}

The requirement of distinctness, uniformity and stability are assessed on the basis of characteristics. The characteristics are a feature of whole plant or part of plant. Such characteristics may be morphological, biochemical, molecular or any other nature. The table of characteristics chosen by experts forms the main part of test guidelines and of DUS testing. In Genetic resources, the term 'characteristic' is known as descriptors (with descriptor states) and describing a plant based on such descriptor is known as 'characterization'.

\section{Morphological characterization}

This is based on botanical or morphological descriptors or characters of the plant or plant part. Qualitative characteristics: Truly qualitative characteristics show discrete discontinuous states and are stable, heritable and uniformly expressed in all environments (shape, flower color, etc).

Pseudo-qualitative characteristics: Here, the range of expression is at least partly continuous varying in more than one dimension. In some cases, intermediate states of expression such as 'weakly expressed' are included between 'absent' and 'strongly expressed' (Pubescence, Pigmentation, etc.). Qualitative characteristics are assessed visually while quantitative characteristics are usually measured. The following types of assessments are recommended:

VG: Visual assessment by a single observation of a group of plants or parts of plant.

VS: Visual assessment by observation of individual plants or parts or plants.

MG: Measurement by a single observation of a group of plants or parts of plant.

MS: Measurement of a number of individual plants or parts or plant.

Grouping characteristics can be universally used, either individually or collectively, for grouping the similar varieties.

These characteristics are considered to be most reliable in distinguishing or discriminating varieties.

Biochemical characterization: Isozyme based descriptors have been widely used for identification of crop varieties because of their reliability (Smith and Smith, 1992; Cooke, 1995). 
Table.1 Classification of DUS traits associated with cowpea

\begin{tabular}{|c|l|c|c|}
\hline S. N0 & DUS traits associated with & Frequency & Percentage \\
\hline $\mathbf{0 1}$ & Seedling stage (Anthocynin coloration) & 01 & 1.08 \\
\hline $\mathbf{0 2}$ & Stem & 02 & 2.16 \\
\hline $\mathbf{0 3}$ & flower & 07 & 7.61 \\
\hline $\mathbf{0 4}$ & Plant & 10 & 10.86 \\
\hline $\mathbf{0 5}$ & Leaf & 13 & 14.44 \\
\hline $\mathbf{0 6}$ & Pod & 18 & 19.56 \\
\hline $\mathbf{0 7}$ & Seed & 23 & 25.00 \\
\hline $\mathbf{0 8}$ & Response to abiotic stress & 04 & 4.35 \\
\hline $\mathbf{0 9}$ & Response to biotic stress (Insect Pests) & 05 & 5.43 \\
\hline $\mathbf{1 0}$ & Response to biotic stress (Diseases) & 09 & 9.78 \\
\hline
\end{tabular}

Table.2 Proposed cowpea crop descriptors

\begin{tabular}{|c|c|c|c|c|}
\hline S. No & Characters & Status & Code & Adopted from \\
\hline \multirow[t]{2}{*}{1} & \multirow{2}{*}{$\begin{array}{l}\text { Seedling Anthocyanin } \\
\text { coloration }\end{array}$} & Absent & 0 & \multirow[t]{2}{*}{ IITA, Nigeria } \\
\hline & & Present & 1 & \\
\hline \multirow[t]{3}{*}{2} & \multirow[t]{3}{*}{ Stem color } & Purple & 1 & \multirow[t]{3}{*}{ IITA, Nigeria } \\
\hline & & Semi purple & 2 & \\
\hline & & Green & 3 & \\
\hline \multirow[t]{3}{*}{3} & \multirow[t]{3}{*}{ Stem hairiness } & Non-hairy & 3 & \multirow[t]{3}{*}{ IITA, Nigeria } \\
\hline & & Sparsely hairy & 5 & \\
\hline & & Hairy & 7 & \\
\hline \multirow[t]{3}{*}{4} & \multirow[t]{3}{*}{ Days to first flowering } & Early $(<35)$ & 1 & \multirow{3}{*}{$\begin{array}{l}\text { Cowpea catalogue, NBPGR, New } \\
\text { Delhi, India }\end{array}$} \\
\hline & & Medium (35-40) & 2 & \\
\hline & & Late $(>40)$ & 3 & \\
\hline \multirow[t]{3}{*}{5} & \multirow[t]{3}{*}{ Days to $50 \%$ flowering } & Early (48) & 1 & \multirow{3}{*}{$\begin{array}{l}\text { Cowpea catalogue, NBPGR, New } \\
\text { Delhi, India }\end{array}$} \\
\hline & & Medium (48-52) & 2 & \\
\hline & & Late $(>52)$ & 3 & \\
\hline \multirow[t]{3}{*}{6} & \multirow{3}{*}{$\begin{array}{l}\text { Plant height (height of } \\
\text { main stem when } \\
\text { supported) }\end{array}$} & Short $(<55)$ & 1 & \multirow[t]{3}{*}{ IITA, Nigeria } \\
\hline & & Medium (55-65) & 2 & \\
\hline & & Long $(>65)$ & 3 & \\
\hline \multirow[t]{3}{*}{7} & \multirow[t]{3}{*}{ No. days to maturity } & Early $(<75)$ & 1 & \multirow[t]{3}{*}{ IITA, Nigeria } \\
\hline & & Medium (75-80) & 2 & \\
\hline & & Late $(>80)$ & 3 & \\
\hline \multirow[t]{4}{*}{8} & \multirow[t]{4}{*}{ Plant pigmentation } & $\begin{array}{l}\text { Moderate on base and tip of } \\
\text { petioles }\end{array}$ & 1 & \multirow[t]{4}{*}{ IITA, Nigeria } \\
\hline & & Extensive & 2 & \\
\hline & & None or Very pale & 3 & \\
\hline & & Almost Solid & 4 & \\
\hline \multirow[t]{2}{*}{9} & \multirow[t]{2}{*}{ Plant growth habit } & Prostrate & 1 & \multirow{2}{*}{$\begin{array}{l}\text { Cowpea catalogue, NBPGR, New } \\
\text { Delhi, India }\end{array}$} \\
\hline & & Semi prostrate & 2 & \\
\hline
\end{tabular}




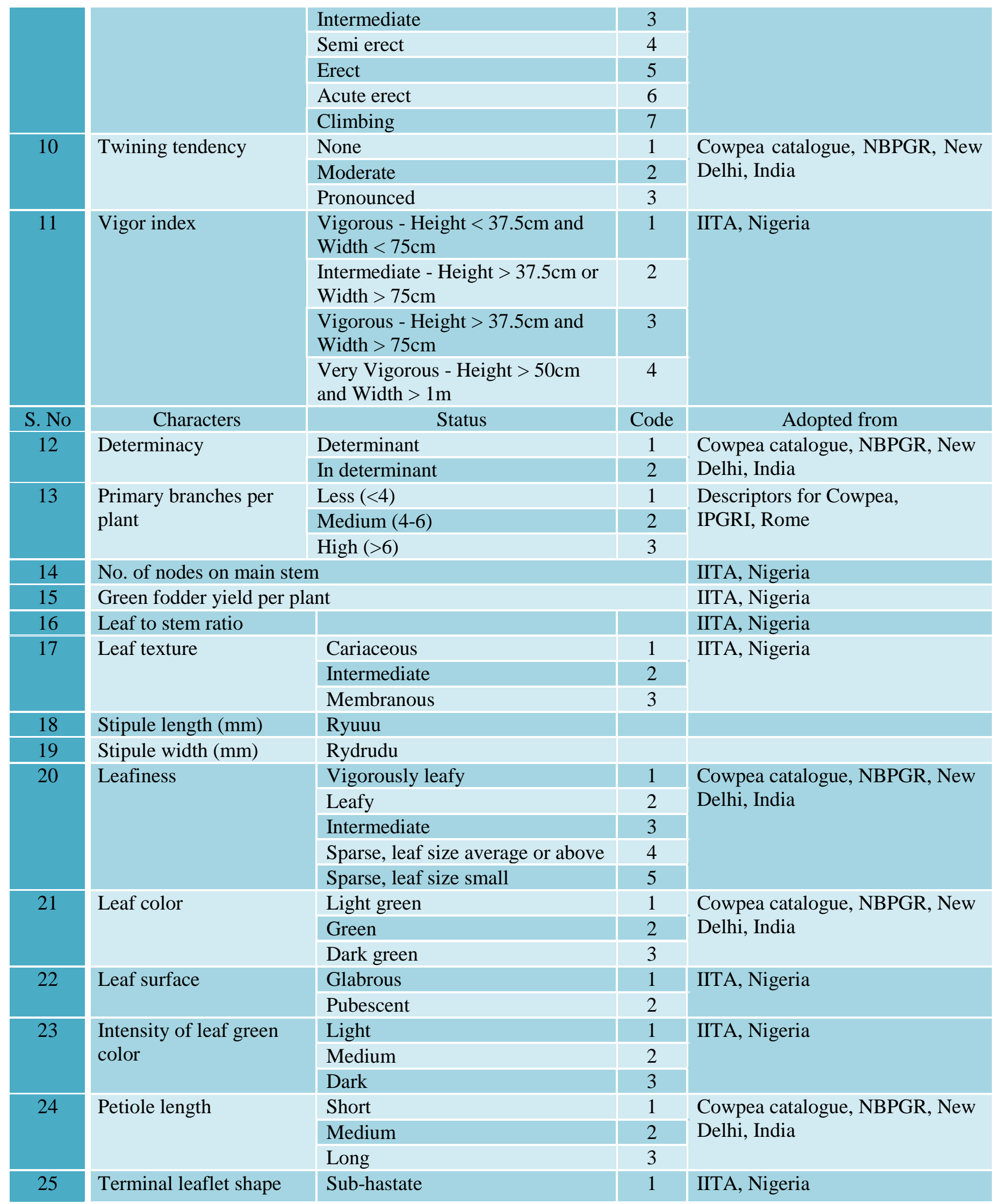




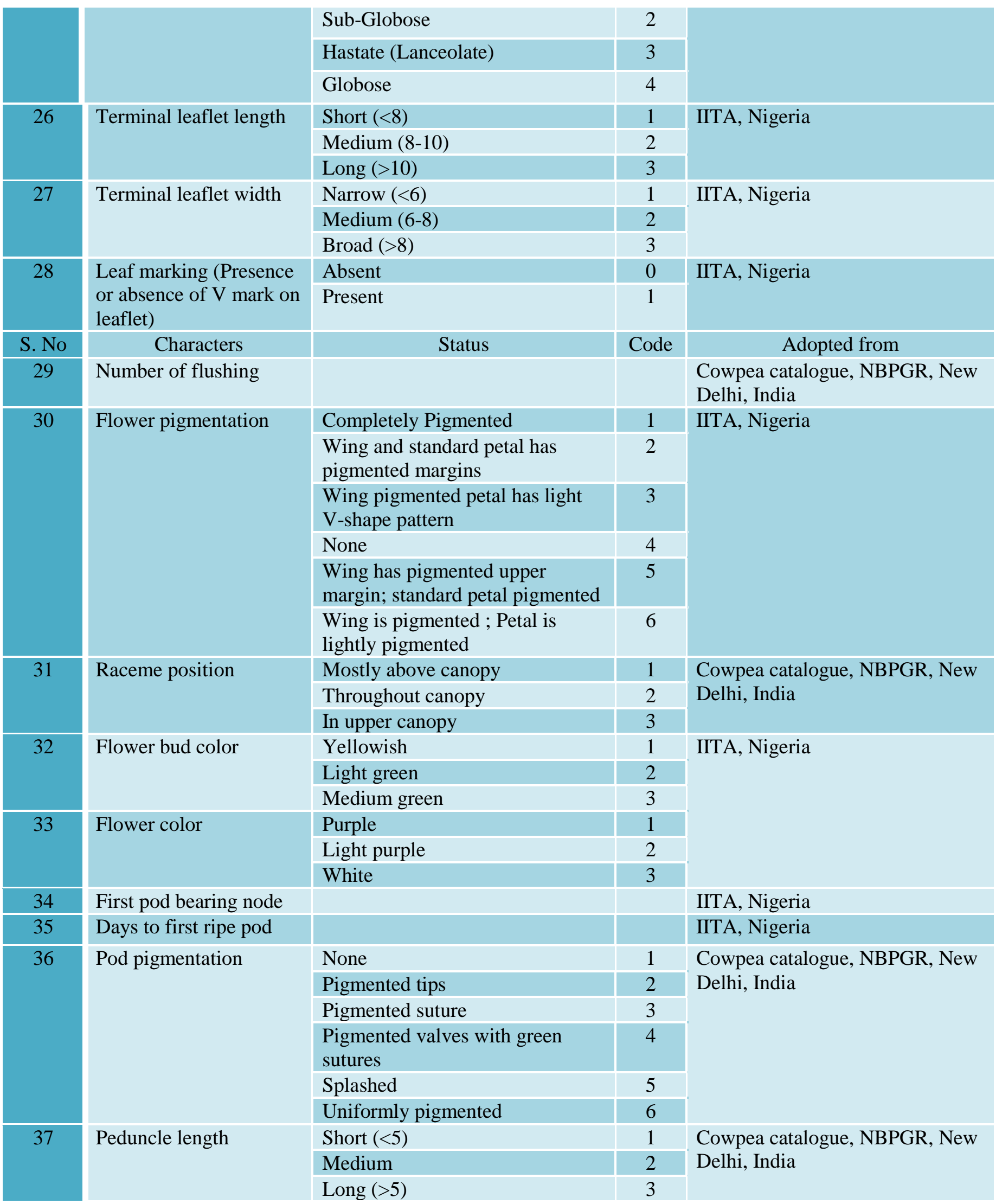




\begin{tabular}{|c|c|c|c|c|}
\hline \multirow[t]{3}{*}{38} & \multirow{3}{*}{$\begin{array}{l}\text { No. of pods per } \\
\text { Peduncle }\end{array}$} & Less $(<2)$ & 1 & \multirow[t]{3}{*}{ IITA, Nigeria } \\
\hline & & Moderate (2-3) & 2 & \\
\hline & & $\operatorname{High}(>4)$ & 3 & \\
\hline \multirow[t]{3}{*}{39} & \multirow[t]{3}{*}{ No. pods per plant } & Low $(<15)$ & 1 & \multirow[t]{3}{*}{ IITA, Nigeria } \\
\hline & & Medium (15-20) & 2 & \\
\hline & & High $(>20)$ & 3 & \\
\hline \multirow[t]{3}{*}{40} & \multirow{3}{*}{$\begin{array}{l}\text { Pod attachment to } \\
\text { peduncle }\end{array}$} & Erect & 1 & \multirow{3}{*}{$\begin{array}{l}\text { Cowpea catalogue, NBPGR, New } \\
\text { Delhi, India }\end{array}$} \\
\hline & & $\begin{array}{l}\text { Angle of 30-90 o down from } \\
\text { erect }\end{array}$ & 2 & \\
\hline & & Pendent & 3 & \\
\hline S. No & Characters & Status & Code & Adopted from \\
\hline \multirow[t]{3}{*}{41} & \multirow{3}{*}{$\begin{array}{l}\text { Number of Clusters per } \\
\text { plant }\end{array}$} & High $(>11)$ & 1 & \multirow[t]{3}{*}{ IITA, Nigeria } \\
\hline & & Medium (9-11) & 2 & \\
\hline & & low $(<9)$ & 3 & \\
\hline \multirow[t]{3}{*}{42} & \multirow[t]{3}{*}{ Pod shape } & Crescent & 1 & \multirow[t]{3}{*}{ IITA, Nigeria } \\
\hline & & Erect & 2 & \\
\hline & & coiled & 3 & \\
\hline \multirow[t]{3}{*}{43} & \multirow[t]{3}{*}{ Pod length } & Long $(>10)$ & 1 & \multirow[t]{3}{*}{ IITA, Nigeria } \\
\hline & & Medium (8-10) & 2 & \\
\hline & & Short $(<10)$ & 3 & \\
\hline 44 & \multicolumn{3}{|l|}{ Pod width } & IITA, Nigeria \\
\hline 45 & \multicolumn{3}{|l|}{ Number of locules per pod } & IITA, Nigeria \\
\hline \multirow[t]{4}{*}{46} & \multirow[t]{4}{*}{ Pod curvature } & Straight & 1 & \multirow[t]{4}{*}{ IITA, Nigeria } \\
\hline & & Curved & 2 & \\
\hline & & Slightly curved & 3 & \\
\hline & & Coiled & 4 & \\
\hline \multirow[t]{4}{*}{47} & Immature pod color & Green & 1 & IITA, Nigeria \\
\hline & & Dark green & 2 & \\
\hline & & Light green & 3 & \\
\hline & & Purple green & 4 & \\
\hline 48 & Pod color at maturity & Yellow & 1 & IITA, Nigeria \\
\hline & & Brown & 2 & \\
\hline 49 & Pod twisting & Absent & 0 & IITA, Nigeria \\
\hline & & Present & 1 & \\
\hline 50 & Texture of pod surface & Smooth & 1 & IITA, Nigeria \\
\hline & & Moderately rough & 2 & \\
\hline & & Very rough & 3 & \\
\hline 51 & Extent of seed crowding & Non Crowder & 0 & IITA, Nigeria \\
\hline & in pod & Semi Crowder & 1 & \\
\hline & & Extreme Crowder & 2 & \\
\hline 52 & $\begin{array}{l}\text { Number of seeds per } \\
\text { pod }\end{array}$ & $\begin{array}{l}\text { Medium (14-18), Low, }(<14) \text {, } \\
\text { high }(>18)\end{array}$ & $1,2,3$ & $\begin{array}{l}\text { Descriptors for Cowpea, } \\
\text { IPGRI, Rome }\end{array}$ \\
\hline 53 & Pod wall thickness & Thin & 3 & IITA, Nigeria \\
\hline & & Intermediate & 5 & \\
\hline & & Thick & 7 & \\
\hline 54 & Eye pattern & Watson group & 1 & IITA, Nigeria \\
\hline & & Holstein group & 2 & \\
\hline
\end{tabular}




\begin{tabular}{|c|c|c|c|c|}
\hline & & Small eye & 3 & \\
\hline \multirow{6}{*}{55} & \multirow[t]{6}{*}{ Eye color } & Narrow eye & 1 & \multirow{6}{*}{ IITA, Nigeria } \\
\hline & & Kabba eye & 2 & \\
\hline & & Tan and Brown & 3 & \\
\hline & & Red & 4 & \\
\hline & & white, cream, brown splash & 5 & \\
\hline & & gray & 6 & \\
\hline S. No & Characters & Status & Code & Adopted from \\
\hline \multirow[t]{2}{*}{56} & \multirow[t]{2}{*}{ Testa texture } & Smooth & 1 & \multirow[t]{2}{*}{ IITA, Nigeria } \\
\hline & & Wrinkled & 2 & \\
\hline \multirow[t]{9}{*}{57} & \multirow[t]{9}{*}{ Seed size } & $1(810 \mathrm{mg} / 10$ seeds $)$ & 1 & \multirow[t]{9}{*}{ IITA, Nigeria } \\
\hline & & $2(1400 \mathrm{mg} / 10$ seeds $)$ & 2 & \\
\hline & & $3(472 \mathrm{mg} / 10$ seeds $)$ & 3 & \\
\hline & & $4(2150 \mathrm{mg} / 10$ seeds $)$ & 4 & \\
\hline & & $5(48 \mathrm{mg} / 10$ seeds $)$ & 5 & \\
\hline & & $6(189 \mathrm{mg} / 10$ seeds $)$ & 6 & \\
\hline & & 7 (2880mg/10 seeds) & 7 & \\
\hline & & 8 (117mg/10 seeds) & 8 & \\
\hline & & $9(3370 \mathrm{mg} / 10$ seeds $)$ & 9 & \\
\hline \multirow[t]{5}{*}{58} & \multirow[t]{5}{*}{ Seed length } & Very short & 1 & \multirow[t]{5}{*}{ IITA, Nigeria } \\
\hline & & Short & 2 & \\
\hline & & Intermediate & 3 & \\
\hline & & Long & 4 & \\
\hline & & Very long & 5 & \\
\hline \multirow[t]{3}{*}{59} & \multirow[t]{3}{*}{ Seed thickness } & Thick & 1 & \multirow[t]{3}{*}{ IITA, Nigeria } \\
\hline & & Flat & 2 & \\
\hline & & Very flat & 3 & \\
\hline \multirow[t]{5}{*}{60} & \multirow[t]{5}{*}{ Seed Shape } & Kidney & 1 & \multirow{5}{*}{$\begin{array}{l}\text { Cowpea catalogue, NBPGR, New } \\
\text { Delhi }\end{array}$} \\
\hline & & Ovoid & 2 & \\
\hline & & Crowdege & 3 & \\
\hline & & Globose & 4 & \\
\hline & & Rhomboid & 5 & \\
\hline \multirow[t]{6}{*}{61} & \multirow{6}{*}{$\begin{array}{l}\text { Seed color (Main color- } \\
\text { color of largest area of } \\
\text { seed) }\end{array}$} & White & 1 & \multirow[t]{6}{*}{ IITA, Nigeria } \\
\hline & & Light yellow & 2 & \\
\hline & & Brown & 3 & \\
\hline & & Reddish brown & 4 & \\
\hline & & Purplish brown & 5 & \\
\hline & & Black & 6 & \\
\hline \multirow[t]{2}{*}{62} & \multirow[t]{2}{*}{ Seed Secondary color } & Absent & 0 & IITA, Nigeria \\
\hline & & Present & 1 & \\
\hline 63 & Seed secondary color & Brown & 1 & IITA, Nigeria \\
\hline & & Reddish brown & 2 & \\
\hline & & Purplish brown & 3 & \\
\hline & & Black & 4 & \\
\hline 64 & Pattern of seed & Around hilum & 1 & IITA, Nigeria \\
\hline & secondary color & In veins & 2 & \\
\hline & & Mottled on part of seed & 3 & \\
\hline
\end{tabular}




\begin{tabular}{|c|c|c|c|c|}
\hline & & Mottled on whole seed & 4 & \\
\hline S. No & Characters & Status & Code & Adopted from \\
\hline \multirow[t]{7}{*}{65} & \multirow[t]{7}{*}{ Seed coat color } & $\begin{array}{l}\text { Buff with brown spot around } \\
\text { hilum }\end{array}$ & 1 & \multirow[t]{7}{*}{ IITA, Nigeria } \\
\hline & & $\begin{array}{l}\text { Buff without brown spot around } \\
\text { hilum }\end{array}$ & 2 & \\
\hline & & brown & 3 & \\
\hline & & Dark red & 4 & \\
\hline & & Tan & 5 & \\
\hline & & Black & 6 & \\
\hline & & Peach & 7 & \\
\hline \multirow[t]{3}{*}{66} & \multirow[t]{3}{*}{ Seed coat lusture } & Shiny & 1 & \multirow[t]{3}{*}{ IITA, Nigeria } \\
\hline & & Intermediate & 2 & \\
\hline & & Dull & 3 & \\
\hline \multirow[t]{2}{*}{67} & \multirow[t]{2}{*}{ Splitting of testa } & Absent & 0 & \multirow[t]{2}{*}{ IITA, Nigeria } \\
\hline & & Present & 1 & \\
\hline \multirow[t]{2}{*}{67} & \multirow[t]{2}{*}{ Attachment of Testa } & Testa not firmly attached to seed & 0 & \multirow[t]{2}{*}{ IITA, Nigeria } \\
\hline & & Testa firmly attached to seed & 1 & \\
\hline \multirow[t]{3}{*}{69} & \multirow[t]{3}{*}{ Test weight } & Bold seeded $(>10)$ & 1 & \multirow[t]{3}{*}{ IITA, Nigeria } \\
\hline & & Medium seeded (7-10) & 2 & \\
\hline & & Small seeded $(<7)$ & 3 & \\
\hline \multirow[t]{3}{*}{70} & \multirow[t]{3}{*}{ Market value } & Grain type & 1 & \multirow[t]{3}{*}{ IITA, Nigeria } \\
\hline & & Vegetable type & 2 & \\
\hline & & Fodder type & 3 & \\
\hline \multirow[t]{3}{*}{71} & \multirow[t]{3}{*}{ Shattering } & Non-shottering & 1 & \multirow{3}{*}{$\begin{array}{l}\text { Cowpea catalogue, NBPGR, New } \\
\text { Delhi }\end{array}$} \\
\hline & & Medium Shottering & 2 & \\
\hline & & Highly shottering & 3 & \\
\hline \multirow[t]{3}{*}{72} & \multirow[t]{3}{*}{ Seed yield per plant } & High $(>60)$ & 1 & \multirow{3}{*}{$\begin{array}{l}\text { Descriptors for Cowpea, } \\
\text { IPGRI, Rome }\end{array}$} \\
\hline & & (Medium (30-60) & 2 & \\
\hline & & Low $(<30)$ & 3 & \\
\hline \multirow[t]{4}{*}{73} & \multirow{4}{*}{$\begin{array}{l}\text { Seed yield } \mathrm{Kg} \text { per } \\
\text { Hectare }\end{array}$} & Low $: \leq 1100$ & 1 & IITA, Nigeria \\
\hline & & Moderate : 1100 - 1600 & 2 & \\
\hline & & High : $1600-2000$ & 3 & \\
\hline & & Very high : > 2000 & 4 & \\
\hline 74 & Incidence of cowpea & None & 0 & IITA, Nigeria \\
\hline & yellow mosaic virus & Green mottle & 1 & \\
\hline & & Yellow Mosaic & 2 & \\
\hline S. No & Characters & Status & Code & Adopted from \\
\hline 75 & Capacity for re-growth & Present/Absent & & IITA, Nigeria \\
\hline 76 & Low temperature & Low Susceptibility & 3 & IITA, Nigeria \\
\hline & & Medium Susceptibility & 5 & \\
\hline & & High Susceptibility & 7 & \\
\hline 77 & High Temperature & Low Susceptibility & 3 & IITA, Nigeria \\
\hline & & Medium Susceptibility & 5 & \\
\hline
\end{tabular}




\begin{tabular}{|c|c|c|c|c|}
\hline & & High Susceptibility & 7 & \\
\hline \multirow[t]{3}{*}{78} & \multirow[t]{3}{*}{ Drought } & Low Susceptibility & 3 & \multirow[t]{3}{*}{ IITA, Nigeria } \\
\hline & & Medium Susceptibility & 5 & \\
\hline & & High Susceptibility & 7 & \\
\hline \multirow[t]{3}{*}{79} & \multirow[t]{3}{*}{ High Soil Moisture } & Low Susceptibility & 3 & \multirow[t]{3}{*}{ IITA, Nigeria } \\
\hline & & Medium Susceptibility & 5 & \\
\hline & & High Susceptibility & 7 & \\
\hline \multirow[t]{3}{*}{80} & \multirow[t]{3}{*}{ Coried bugs } & Low Susceptibility & 3 & \multirow[t]{3}{*}{ IITA, Nigeria } \\
\hline & & Medium Susceptibility & 5 & \\
\hline & & High Susceptibility & 7 & \\
\hline \multirow[t]{3}{*}{81} & \multirow[t]{3}{*}{ Cowpea aphid } & Low Susceptibility & 3 & \multirow[t]{3}{*}{ IITA, Nigeria } \\
\hline & & Medium Susceptibility & 5 & \\
\hline & & High Susceptibility & 7 & \\
\hline \multirow[t]{3}{*}{82} & \multirow[t]{3}{*}{ Pod borer } & Low Susceptibility & 3 & \multirow[t]{3}{*}{ IITA, Nigeria } \\
\hline & & Medium Susceptibility & 5 & \\
\hline & & High Susceptibility & 7 & \\
\hline \multirow[t]{3}{*}{83} & \multirow[t]{3}{*}{ African bollworm } & Low Susceptibility & 3 & \multirow[t]{3}{*}{ IITA, Nigeria } \\
\hline & & Medium Susceptibility & 5 & \\
\hline & & High Susceptibility & 7 & \\
\hline S. No & Characters & Status & Code & Adopted from \\
\hline \multirow[t]{3}{*}{84} & \multirow[t]{3}{*}{ Cowpea storage weevil } & Low Susceptibility & 3 & \multirow[t]{3}{*}{ IITA, Nigeria } \\
\hline & & Medium Susceptibility & 5 & \\
\hline & & High Susceptibility & 7 & \\
\hline \multirow[t]{3}{*}{85} & \multirow[t]{3}{*}{ Ascochyta blight } & Low Susceptibility & 3 & IITA, Nigeria \\
\hline & & Medium Susceptibility & 5 & \\
\hline & & High Susceptibility & 7 & \\
\hline 86 & Powdery mildew & Low Susceptibility & 3 & IITA, Nigeria \\
\hline & & Medium Susceptibility & 5 & \\
\hline & & High Susceptibility & 7 & \\
\hline 87 & Fusarium wilt & Low Susceptibility & 3 & IITA, Nigeria \\
\hline & & Medium Susceptibility & 5 & \\
\hline & & High Susceptibility & 7 & \\
\hline 88 & Pythium stem rot & Low Susceptibility & 3 & IITA, Nigeria \\
\hline & & Medium Susceptibility & 5 & \\
\hline & & High Susceptibility & 7 & \\
\hline 89 & Sclerotium stem rot & Low Susceptibility & 3 & IITA, Nigeria \\
\hline & & Medium Susceptibility & 5 & \\
\hline & & High Susceptibility & 7 & \\
\hline 90 & Brown rust & Low Susceptibility & 3 & IITA, Nigeria \\
\hline & & Medium Susceptibility & 5 & \\
\hline & & High Susceptibility & 7 & \\
\hline 91 & Verticillium wilt & Low Susceptibility & 3 & IITA, Nigeria \\
\hline & & Medium Susceptibility & 5 & \\
\hline & & High Susceptibility & 7 & \\
\hline 92 & Bacterial light and & Low Susceptibility & 3 & IITA, Nigeria \\
\hline & canker & Medium Susceptibility & 5 & \\
\hline & & High Susceptibility & 7 & \\
\hline
\end{tabular}


Table.3 Recommendable quality tests

\begin{tabular}{|l|l|l|}
\hline S. No. & Nutritional Quality test & Status \\
\hline 1 & $\begin{array}{l}\text { Seed protein Content } \\
\text { Lowry et al., (1951) }\end{array}$ & $\begin{array}{l}\text { Low }(<20 \%) \\
\text { Medium }(20-30 \%) \\
\text { High }(>30 \%)\end{array}$ \\
\hline Chemical tests & $\begin{array}{l}\text { Negative } \\
\text { KOH-bleach test } \\
\text { (McDonald-MB Jr., 1985) }\end{array}$ & $\begin{array}{l}\text { Brownish yellow } \\
\text { Light yellow }\end{array}$ \\
\hline 2 & Light yellow \\
\hline 3 & Phenol test (Walls, 1965) & $\begin{array}{l}\text { Negative } \\
\text { Grey }\end{array}$ \\
\hline 4 & $\begin{array}{l}\text { Ferrous sulphate test } \\
\text { (Kumar et al.,1995) }\end{array}$ & $\begin{array}{l}\text { Dark brown } \\
\text { Negative }\end{array}$ \\
\hline 5 & $\begin{array}{l}\text { Sodium hydroxide test } \\
\text { (McDonald-MB Jr.,1985) }\end{array}$ \\
\hline & Dark brown \\
& & Yellow \\
\hline & Light yellow \\
& & Brownish yellow \\
& & Light brown \\
\hline & Reddish brown \\
\hline
\end{tabular}

UPOV has also included electrophoresis of iso-enzymes in maize, soybean, sunflower and of seed proteins in barley, wheat as additional characters for establishing distinctness of varieties. Selection of an appropriate electrophoresis technique provides a potential tool for variety identification, DUS test or grouping of varieties.

Molecular characterization: Biotechnology has widened the possibilities for applying such technologies to the problem of characterization, varietal identification and protection (Smith, 1995). At present, molecular markers are not being used in DUS testing anywhere, but they are reliable, fast and cost effective to discriminate an EDV (essentially derived variety). Data from these methods may help resolve disputes on identity of germplasm or hybrids (Santhy et al., 2003).

\section{DUS test design}

The use of experimental design with respect to the number of growing cycles, lay out of the trial, number of plants to be examined and method of observation is largely determined by the number and nature of varieties to be examined in a particular trial.

In DUS trials, because of the presence of only one treatment factor (variety), the following designs are used

Completely Randomized Design- if total number of test varieties is small. Several varieties are examined in a number of replications.

Randomized complete Block Design- the number of plots per block equals the number of varieties and all varieties are placed in each 
block. The advantage is that standard deviation between plots does not contain variation due to difference in blocks.

Randomized incomplete Block Design- in case of large number of varieties. Here, the number of plots per block is less than the number of varieties.

In Poland, performed analysis of variance of the results of experiment concerning seven characters in pea varieties showed that randomized complete block and completely randomized designs were more effective than incomplete block (Pilarczyk, 1999).

\section{Reference collection}

To test whether a candidate variety meets the DUS criteria, it is compared with varieties whose existence is a matter of common knowledge. To satisfy the requirement of distinctness, a candidate variety must be clearly distinguishable from all other existing varieties. These varieties are called the varieties of common knowledge, which includes:

\section{Protected varieties}

Varieties listed in official register

Varieties, subject of an application for protection

Varieties listed in any commercial document in which varieties are offered for marketing in its territory as propagating material, especially where there is no official registration system.

Ecotypes and land races

Publicly available varieties within plant germplasm collection (genetic resources, old varieties, etc.
Literature search regarding the DUS characterization in cowpea published in peer reviewed journals and crop descriptors available in the organizations like NBPGR, New Delhi, IPGRI, Rome and IITA, Nigeria has provided an idea and list of most appropriate descriptors to characterize germplasm resources in terms of distinctness, unity, stability and novelty (DUSN). Based insight review, we classified plant parameters in to ten different categories considering leaf, stem, habitat, flower, pod and seed characteristics (Table 1).

Further, DUS traits under each category were developed according to the mode of phenotypic expression of the character in question and based on the response of germplasm to biotic and abiotic stress. The present review proposes to consider the newly identified parameters as newly proposed DUS traits for morphological characterization of cowpea germplasm (Table 2).

The distinctness among the germplasm may also be determined at biochemical level, as we know about the isozyme and allozyme biochemical marker. The prevalence of isozyme allozyme biochemical markers informs or shows a clue regarding characterization or establishing the distinctness at biochemical level. In this regard, a vast literature search related biochemical aspects of cowpea has been made and come out with following six different quality tests (Table 3) to characterize the cowpea germplasm.

\section{Acknowledgement}

A project entitled, DUS characterization of cowpea landraces, germplasm and released varieties for grain and yield traits have been granted by "Protection of Plant Varieties and Farmers Rights Authority" IARI Campus, New Delhi-110 001. Under the project, a 
preliminary literature search has been made to identify and develop new crop descriptors. In this context, a review article has been formulated.

\section{References}

Basavaraj Makanur, V. K. Deshpande S. K. and Vyakaranahal, B. S. 2013, Characterization of cowpea genotypes based on Quantitative descriptors, The Bioscan, 8 (4): 1183-1188,

Cooke R.J.1995. Review: Gel electrophoresis for the identification of plant varieties. Journal of Chromatography 698:281299.

Davis, D. W., Oelke, E. A., Oplinger, E. S., Doll, J. D., Hanson, C.V., Putnam, D. H., 1991, Cowpea, Alternative Field Crops Manual.

Egbadzor, Kenneth, F., Ofori, K., Yeboah, M., Aboagye, Lawrence, M., OpokuAgyeman, Michael O; Danquah, Eric, Y., Offei. and Samuel K., 2014, Diversity in 113 cowpea (Vigna unguiculata (L) Walp) accessions assessed with 458 SNP markers, Springer Plus, 3: 541.

Kumar, A., Chowdhary, R. K., Kapoor, R. L. and Dahiya, O.S., (1995), Identification of pearl millet hybrids and their parental lines using seeds and seedling characters, chemical tests and gel electrophoresis. Seed Sci. and Technol., 23: 21-32.

Lowry, O.H., Rosebrough, N.J., Farr, A.L and Randall, R.J. 1951. Protein measurement with folin-phenol reagent. Journal of Biochemistry. 193: 265-275.

Mc Donald MB Jr. 1985. AOSA cultivar purity subcommittee report. AOSA Newsletter 59(1):40-57.

National Research Council, 2006, "Cowpea". Lost Crops of Africa: Volume II: Vegetables. Washington DC: The National Academies Press., 104-117.
Padulosil, S. and Ng, N. Q., 1997, Origin, taxonomy, and morphology of Vigna unguiculata (L.) Walp.". In Singh, B. B.; Mohan, D. R.; Dashiell, K. E.; Jackai, L. E. N. Advances in Cowpea Research(PDF). Ibadan, Nigeria: International Institute of Tropical Agriculture and Japan International Research Center for Agricultural Sciences.

Pilarczyk W.1999. On efficiency of resolvable incomplete block design in DUS trials on French bean varieties. UPOV TWC/17/2.

Pottorff, M., Ehlers., Jeffrey D., Fatokun, C., Roberts., Philip, A. and Timothy J., 2012, Leaf morphology in Cowpea (Vigna unguiculata (L.) Walp) QTL analysis, physical mapping and identifying a candidate gene using synteny with model legume species, BMC Genomics. 13: 234.

Rawal, Kanti M., 1975, Natural hybridization among wild, weedy and cultivated Vigna unguiculata (L.) Walp, Euphytica, 24 (3): 699-707.

Rupinder Kaur, A. K., Toor., Geeta Bassi. and Bains, T. S., 2017, Characterization of Mungbean (Vigna radiata L. Wilczek) Varieties using Morphological and Molecular Descriptors Int.J.Curr. Microbiol.App.Sci., 6(6): 1609-1618.

Santhy, V., Mohapatra, T., Dadlani, M., Sharma, S. P. and Sharma, R. P., 2000, DNA markers for testing distinctness of rice varieties. Plant vars and seeds, 13:141-148.

Sarutayophat, T., Nualsri, C., Santipracha, Q. and Saereeprasert, V., 2007, Characterization and genetic relatedness among 37 yard long bean and cowpea accessions based on morphological characters and RAPD analysis. Songklanakarin J. Sci. Technol., 29(3): 591-600. 
Sheahan, C. M. 2012, Plant guide for cowpea (Vigna unguiculata), USDA-Natural Resources Conservation Service, Cape May Plant Materials Center, Cape May, NJ.

Smith J.S.C., 1995, Identification of cultivate varieties by nucleotide analysis. In Identification of food grain varieties, Wrigley C.W. (ed.). AACC, USA.

Smith O.S and Smith, J. S. C., 1992, Measurement of genetic diversity among maize hybrids. A comparison of isozyme, RFLP, pedigree and heterosis data. Maydica, 37:53-60.

Stoilova, T. and Pereira, G. 2013, Assessment of the genetic diversity in a germplasm collection of cowpea (Vigna unguiculata (L.) Walp.) using morphological traits. African J. Agric. Res. 8(2): 208-215.

Sunil Kumar., Dalbir Phogat. and Nabin Bhusal., 2015, Characterization of elite forage cowpea genotypes for Various DUS traits. Forage res., 40 (4): 232236.

Walls, F.M., 1965, A standardized phenol method for testing wheat for varietal purity. Handbook of Seed Testing. Contribution No. 28, Assoc. of Official Seed Analysts.

\section{How to cite this article:}

Sanjeev K. Deshpande, B.R. Mani, S.A. Desai, T.K. Nagarathna and Hanchinal, R.R. 2018. Review on Characterization of Cowpea Germplasm in Terms of Distinctness, Uniformity, Stability and Novelty for Morphological, Quality and Yield Attributing Parameters. Int.J.Curr.Microbiol.App.Sci. 7(06): 1124-1139. doi: https://doi.org/10.20546/ijcmas.2018.706.134 